\title{
Use of High-Flow Continuous Renal Replacement Therapy with Citrate Anticoagulation to Control Intracranial Pressure by Maintaining Hypernatremia in a Patient with Acute Brain Injury and Renal Failure
}

\author{
Joshua E. Medow, MD; Shalin R. Sanghvi, MD; and R. Michael Hofmann, MD
}

Traumatic brain injury and intracranial hypertension often require treatment to optimize patient outcome. There are a variety of complex medical conditions that can preclude standard approaches to the treatment of intracranial hypertension. We describe a case where a novel approach using continuous dialysis with trisodium citrate was used to optimize the outcome of a young male with acute renal failure and acute respiratory distress syndrome in the setting of acute traumatic brain injury.

Keywords: Intracranial pressure; Renal failure; Dialysis; CRRT; CVVH

Corresponding Author:

Joshua E. Medow, MD

Director Neurocritical Care

Asst Prof Neurosurgery \& Biomedical Engineering

University of Wisconsin

Neurosurgery \& Biomedical Engineering

K4/822 CSC

600 Highland Ave

Madison, WI 53792

Tel: (608) 609-9278

Fax: (608) 263-1728

Email:medow@neurosurgery.wisc.edu

Received: March 13,2014

Revised: August 6, 2014

Accepted: August 13,2014

doi: $10.3121 / \mathrm{cmr} .2014 .1238$

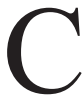
ontinuous renal replacement therapy (CRRT) has grown in popularity as a method of providing continuous dialytic therapy to critically ill patients. It provides excellent clearance of solute while avoiding the risk of systemic hypotension and dramatic changes in intracranial pressure (ICP). There is still controversy regarding, optimal modality if hemofiltration versus hemodialysis offers an advantage over the other in the setting of acute kidney injury (AKI). Initial trials suggested high flow $(>35 \mathrm{~mL} / \mathrm{kg} / \mathrm{h})$ CRRT improved patient outcomes in the setting of AKI, ${ }^{1}$ but more recent studies demonstrated no survival advantage over more moderate dosing of CRRT $(20 \mathrm{~mL} / \mathrm{kg} / \mathrm{h}){ }^{2}$ Citrate anticoagulation prolongs filter life in continuous systems without increasing the risk of systemic bleeding. However, studies implementing high flow CRRT with citrate anticoagulation are limited.

Patients with severe acute traumatic brain injury (TBI) are complex and difficult to manage in the intensive care unit (ICU). The focus of care is to maintain cerebral perfusion pressure (CPP), recognizing that there is a critical relationship between mean arterial pressure (MAP) and ICP. CPP is defined as MAP - ICP, and the goal is to maintain CPP $>60 \mathrm{~mm} \mathrm{Hg}$ at all times. CPP must be maintained to prevent infarction/secondary brain injury. Many therapies are targeted at acutely increasing the serum osmolality to decrease the ICP. Recent literature has advocated the use of intravenous (IV) hypertonic sodium over mannitol to control brain edema following acute TBI.,

Patients may develop AKI after acute TBI, which is frequently exacerbated by the high osmolality often seen with excessive use of osmotic agents such as 
mannitol. ${ }^{5}$ There are many additional challenges when treating TBI patients that develop severe AKI requiring renal replacement, including rapid shifts in osmolality, hemodynamic derangements, increased systemic coagulation, worsening cerebral edema, and intra-cranial acidosis. ${ }^{6}$ CRRT has been advocated in patients with elevated ICP, as it has been shown to result in greater ICP stability. ${ }^{6}$

We describe a case of AKI and severe brain injury where high-flow CRRT with citrate anticoagulation was used to maintain hypernatremia (due to the trisodium component of the citrate) and a euvolemic intravascular volume until there was resolution of the patient's elevated ICP and renal compromise, while also serving to provide excellent circuit anticoagulation.

\section{Case Report}

A previously healthy caucasian man, aged 18 years, sustained a closed head injury after falling out of a golf cart and striking his head on a tree. An initial computed tomography (CT) scan demonstrated a left subdural hematoma with moderate edema, bilateral contusions, and diffuse traumatic subarachnoid hemorrhage, but no intraventricular blood or hydrocephalus. Chest CT scan also showed moderate to severe pulmonary contusions. An ICP monitor was placed, showing ICPs in excess of $60 \mathrm{~mm} \mathrm{Hg}$. Initial therapies to lower ICP included reverse Trendelenburg, ventriculostomy, and 75 grams of IV mannitol. Based on the severe degree of intracranial injury and worsening clinical pictures, the patient was transferred to the University of Wisconsin Hospital and Clinics for further management.

Upon arrival, the patient was commenced on IV mannitol, and paralytic and sedative drips. An emergent left-sided decompression and frontal lobectomy were performed. At cranial closure, ICP measurements ranged from 7 to 14 $\mathrm{mm} \mathrm{Hg}$ but rose to $60 \mathrm{~mm} \mathrm{Hg}$ over the next 24 hours, requiring barbiturate coma. Over the ensuing days, the patient was maintained in a thiopental-induced coma with IV mannitol boluses every 2 hours. ICP remained elevated but was generally $<25 \mathrm{~mm} \mathrm{Hg}$. Serum osmolality exceeded 350 $\mathrm{mOsm} / \mathrm{L}$ at its peak value prior to switching therapies. Hypertonic saline (3\%) continuous infusion was initiated to maintain a serum sodium level between 155 to $160 \mathrm{mmol} / \mathrm{L}$. ICP remained controlled $(<25 \mathrm{~mm} \mathrm{Hg})$, but oxygenation declined progressively due to volume overload and pulmonary contusion. Seven days after admission, the patient developed ARDS requiring $100 \%$ oxygen and permissive hypercapnia to maximize oxygenation. He concomitantly developed increasing ICP's to $40 \mathrm{~mm} \mathrm{Hg}$. The patient was then cooled to $33^{\circ} \mathrm{C}$. His MAP was maintained to reach a goal cerebral perfusion pressure of $55 \mathrm{~mm} \mathrm{Hg}$. On hospital day 9 the patient developed oliguric AKI.

On hospital day 10, the patient progressed to anuric AKI. CRRT was initiated due to elevated ICP, azotemia, and volume overload in the face of anuric AKI. The patient was

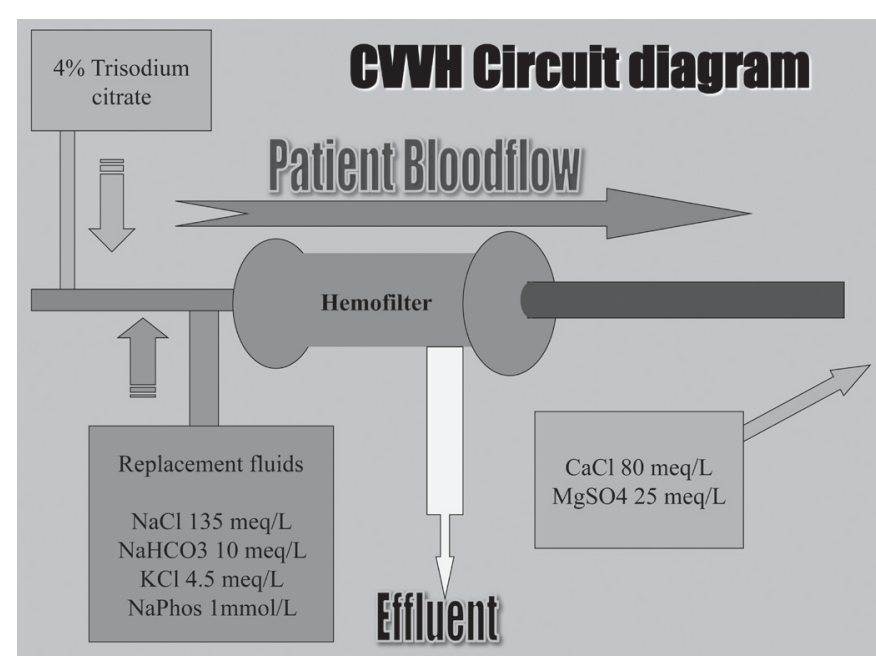

Figure 1. Continuous Veno-Venous Hemofiltration (CVVH) circuit diagram.

initiated on the NxStage System one (NxStage, Lawrence $\mathrm{MA})$ in the continuous veno-venous hemofiltration (CVVH) mode. The target serum sodium was $160 \mathrm{mmol} / \mathrm{L}$ with the goal of maintaining hyperosmolality due to elevated ICP. The blood flow was run at $250 \mathrm{~mL} / \mathrm{min}$ and replacement fluids at $3000 \mathrm{~mL} / \mathrm{h}$ pre-filter. Anticoagulation was achieved by infusing 4\% trisodium citrate (Baxter, Deerfield IL) at 350 $\mathrm{mL} / \mathrm{h}$ immediately after the blood had entered the tubing set from the catheter to optimize anticoagulation in the extracorporeal system. Replacement fluids were infused prefilter and contained $154 \mathrm{mmol} / \mathrm{L}$ of sodium chloride, 4.5 $\mathrm{meq} / \mathrm{L}$ of potassium chloride, $10 \mathrm{meq} / \mathrm{L}$ sodium bicarbonate, and $1.5 \mathrm{mmol} / \mathrm{L}$ of sodium phosphate. Calcium chloride $(80$ $\mathrm{mEq} / \mathrm{L})$ and magnesium sulfate $(25 \mathrm{mEq} / \mathrm{L})$ were added to a liter of normal saline $(154 \mathrm{mEq} / \mathrm{L} \mathrm{Na})$ and were infused via a separate central venous catheter into the systemic circulation at a rate of $180 \mathrm{~mL} / \mathrm{h}(40 \mathrm{mmol} / \mathrm{L})$. Post-filter ionized calcium was maintained at 0.25 to $0.35 \mathrm{mmol} / \mathrm{L}$ while maintaining systemic ionized calcium levels at 1.10 to $1.25 \mathrm{mmol} / \mathrm{L}$ (figure 1). All fluids were compounded by our inpatient pharmacy and modified based on electrolyte changes in the patient's serum by the critical care nephrology team.

After initiating citrate CRRT, it was noted there was a precipitous drop in ICPs that seemed to correlate with the induced hyperosmolarity and decline in the patient's central venous pressure (CVP). The CRRT system was set to maintain a net removal of $200 \mathrm{~mL} / \mathrm{h}$ while maintaining hyperosmolality. The serum sodium was maintained at 160 $\mathrm{mmol} / \mathrm{L}$ until hospital day 16 . At that time the serum sodium was lowered to $155 \mathrm{mmol} / \mathrm{L}$ without significant change in ICP. Utilizing CRRT and monitoring ICP, the serum sodium was gradually lowered to $145 \mathrm{mmol} / \mathrm{L}$ over the next couple of days with no change in ICP.

By hospital day 17 the patient's respiratory status began improving, and he was no longer requiring pressure control ventilation and permissive hypercapnia. His fraction of inspired oxygen (FIO2) requirements decreased. He also began 
making more urine. His renal replacement therapy was discontinued on day 20, as his urine output and function had markedly improved. He required no further renal replacement therapy and was extubated.

The patient was discharged to the rehabilitation medicine service 7 weeks after his injury. He was alert and oriented x 4 with memory intact to events just prior to the accident. He was ambulating with little assistance and was appropriately interactive. His blood urea nitrogen was $17 \mathrm{mg} / \mathrm{dL}$ and creatinine was $0.9 \mathrm{mg} / \mathrm{dL}$. Six months after the accident, the patient completed his high school equivalency with only subtle neuropsychological findings in short term memory capacity and motivation.

\section{Discussion}

CRRT offers several advantages over intermittent hemodialysis in the patient with TBI. These advantages including slower but prolonged total solute removal, preserved hemodynamics, more gradual improvement in acid/base disorders, and decreased vasogenic edema. ${ }^{7,8}$ The management of a patient with severe TBI focuses on lowering ICP and, most importantly, optimizing CPP. Early in the course of brain injury there is marked cerebral edema, which can cause a secondary ischemic injury. Early recognition and treatment can lessen this injury and is the focus of early management of TBI.

Current management strategies to decrease ICP include patient positioning, sedation, hyperosmotic agents, surgical intervention (ventriculostomy and/or craniectomy), normothermia, limited hypothermia, paralytic medications, and barbiturate coma. Recent literature has compared hypertonic sodium to mannitol regarding the ideal hyperosmotic agent. Hypertonic sodium has certain benefits including less renal toxicity, less rebound effect, more pronounced effect on ICP, and longer duration of effect on ICP. ${ }^{3,4,9}$ When the kidneys fail, other osmotic agents enter the vascular supply and tissues at greater concentrations. As an example, urea is generally considered an ineffective osmole. This means that it is able to equilibrate across the vascular and tissue spaces. It does not, however, do this rapidly but rather over time. When urea was used in the treatment of elevated ICP, it worked because it did not equilibrate quickly. Slow equilibration allowed normal kidneys to clear the urea and in doing so, the osmotic diuretic effect was realized. This subsequently caused fluid from the tissues to enter the vascular space, and thus, tissue water decreased as a consequence. In the cranium, this reduction in water resulted in improved ICP. However, this urea sword has two sides. If urea builds up slowly in the vascular system (as seen in renal failure), it is doing so similarly in the tissues. Rapid removal from the vascular compartment, as is seen with hemodialysis (not CRRT), can cause a fluid shift from the vascular space into the tissues, because the tissues have not had time to leach out their stores of urea. Basically, significant increases in tissue osmolality relative to the vascular compartment occur with hemodialysis. This rapid change in osmotic gradient between brain tissue and vascular space leads to cerebral edema, which may cause dialysis equilibrium syndrome. ${ }^{10}$ Early in the process, relative hypotension and headache may be the only warning symptoms. However, if not corrected, severe acute confusion and seizures can ensue. However, in patients with TBI, the early warning signs are often not appreciable due to decreased levels of consciousness, and the secondary increase in ICP can be devastating. Therefore, the slower removal of urea has led to the acceptance that CRRT is the preferred method of renal replacement in those patients with elevated ICP and AKI requiring dialysis. ${ }^{6}$ In this case report, CRRT was used to slowly decrease the patient's expanded extracellular fluid (ECF) state, while both removing solute and hyperosmolality with hypernatremia. By both ECF volume and maintaining hypernatremia, ICP was better controlled. In more gradual dialysis, disequilibrium was avoided.

Although replacement fluids could have been formulated to target a serum sodium of $160 \mathrm{mmol} / \mathrm{L}$ without anticoagulation, we appreciated the risk of frequent clotting of the filter without anticoagulation particularly in post-trauma patients. Given the particularly labile ICPs of our patient with a recent significant trauma, we decided on regional citrate anticoagulation to prolong filter life, avoid systemic anticoagulation, and minimize system "downtime," which may have led to much less effective delivery of the planned CRRT therapy.

Citrate anticoagulation in CRRT has enjoyed increasing popularity, since it provides anticoagulation that exceeds the filter patency of systems maintained with a heparin infusion. ${ }^{11-13}$. The use of citrate anticoagulation has occasionally resulted in severe electrolyte abnormalities including hypernatremia ${ }^{12,14}$ stemming from the large salt load found in trisodium citrate preparations. We used that excess sodium to our advantage in this case as a means to improve ICP and provide anticoagulation. Although often more complex, citrate anticoagulation does offer the distinct advantage of only providing circuit anticoagulation with no increased risk of systemic anticoagulation to the patient. This is of paramount importance in patients with acute TBI who are at high risk of acute secondary intracranial hemorrhage.

We have previously described a method for using citrate anticoagulation in CRRT ${ }^{15}$ and have since adapted it for highflow applications. We appreciated the potential to induce hypernatremia with trisodium citrate through our extensive history of using citrate CRRT. In this case, we felt that we could target a stable level of hypernatremia in order to control the elevated ICPs in our patient. After deciding on a goal sodium level of $160 \mathrm{mmol} / \mathrm{L}$ to control ICP, we modified our usual replacement fluids to achieve that target (figure 2).

Following initiation of the CRRT with citrate anticoagulation, the patient's MAP remained stable at $>80 \mathrm{~mm} \mathrm{Hg}$ and CPP 


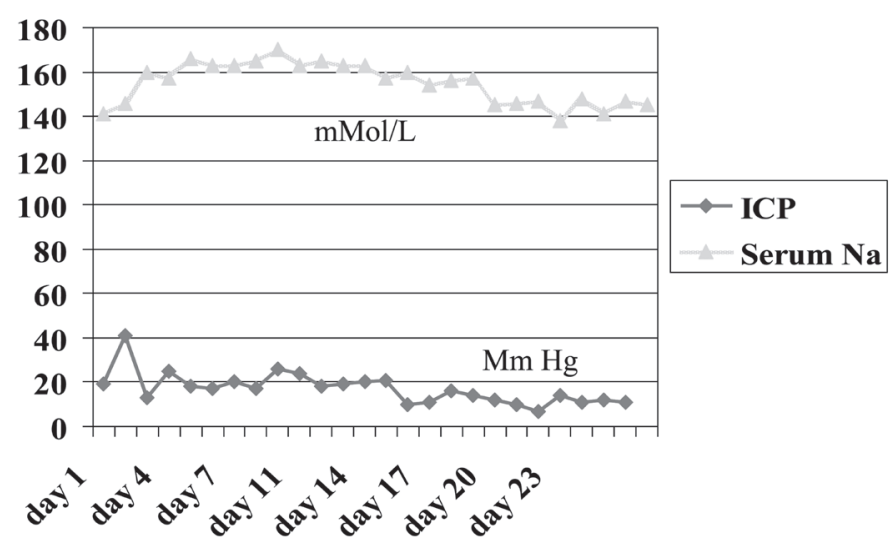

Figure 2. Patient's intracranial pressure (ICP) versus serum Na levels.

$>60 \mathrm{~mm} \mathrm{Hg}$ for the duration of CRRT. The CVP decreased from $17 \mathrm{~mm} \mathrm{Hg}$ to $6 \mathrm{~mm} \mathrm{Hg}$ during CRRT (figure 3). While previous data has shown elevated ICP with initiation of intermittent hemodialysis, ${ }^{10}$ this was not observed in our patient. Other advantages offered by the continuous system included volume removal while maintaining stable CVP (6 to $10 \mathrm{~mm} \mathrm{Hg}$ ), constant solute removal, and maintaining electrolytes as desired by the involved teams. Traditional "continuous" systems have been plagued with down time as well as blood loss secondary to filters clotting. The trisodium citrate anticoagulation offered the major benefit of prolonged filter life without the risk of systemic bleeding. While there have been concerns regarding hypernatremia, alkalosis, and hypocalcemia when using trisodium citrate anticoagulation, the replacement fluid takes into account the metabolic effects and compensates for these derangements. Furthermore, in this case, as hypernatremia was a desired effect, the replacement fluid sodium was increased to achieve the serum sodium goal of $160 \mathrm{mmol} / \mathrm{L}$. There were no complications known due to the CRRT system implemented in this case.

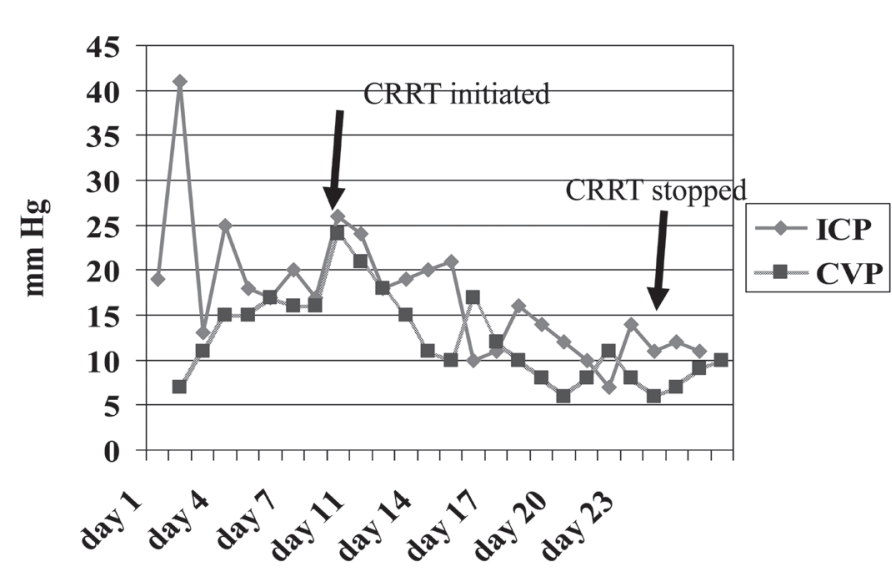

Figure 3. Patient's intracranial pressure (ICP) versus central venous pressure (CVP) during continuous renal replacement therapy (CRRT).

\section{Conclusion}

This is the first described use of trisodium citrate anticoagulation with high-flow CRRT to induce continuous hypernatremia in the setting of TBI with elevated ICP. While there are concerns regarding metabolic derangement secondary to the trisodium citrate, if these are accounted for, trisodium citrate can be used safely and effectively. Furthermore, if the conversion of the anticoagulant to sodium and bicarbonate is appreciated, they can be used to maintain specific electrolyte profiles on these patients. Previous literature has highlighted the use of CRRT for patients with acute brain injury. We suggest considering trisodium citrate regional anticoagulation to induce hypernatremia in patients with AKI and elevated ICP as an alternative to the limited standard therapies offered to this select critically ill patient population.

\section{References}

1. Ronco C, Bellomo R, Homel P, Brendolan A, Dan M, Piccinni P, La Greca G. Effects of different doses in continuous venovenous haemofiltration on outcomes of acute renal failure: a prospective randomised trial. Lancet 2000;356:26-30.

2. VA/NIH Acute Renal Failure Trial Network, Palevsky PM, Zhang JH, O'Connor TZ, Chertow GM, Crowley ST, Choudhury D, Finkel K, Kellum JA, Paganini E, Schein RM, Smith MW, Swanson KM, Thompson BT, Vijayan A, Watnick S, Star RA, Peduzzi P. Intensity of renal support in critically ill patients with acute kidney injury. N Engl J Med 2008;359:7-20.

3. Khanna S, Davis D, Peterson B, Fisher B, Tung H, O'Quigley J, Deutsch R. Use of hypertonic saline in the treatment of severe refractory posttraumatic intracranial hypertension in pediatric traumatic brain injury. Crit Care Med 2000;28:1144-1151.

4. Larive LL, Rhoney DH, Parker D, Jr., Coplin WM, Carhuapoma JR. Introducing hypertonic saline for cerebral edema: an academic center experience. Neurocrit Care 2004;1:435-440.

5. Perez-Perez AJ, Pazos B, Sobrado J, Gonzalez L, Gandara A. Acute renal failure following massive mannitol infusion. Am J Nephrol 2002;22:573-575.

6. Davenport A. Renal replacement therapy in the patient with acute brain injury. Am J Kidney Dis Mar 2001;37:457-466.

7. Davenport A, Will EJ, Davison AM. Continuous vs. intermittent forms of haemofiltration and/or dialysis in the management of acute renal failure in patients with defective cerebral autoregulation at risk of cerebral oedema. Contrib Nephrol 1991;93:225-233.

8. Davenport A, Will EJ, Davison AM. Effect of renal replacement therapy on patients with combined acute renal and fulminant hepatic failure. Kidney Int Suppl 1993;41:S245-S251.

9. Murphy N, Auzinger G, Bernel W, Wendon J. The effect of hypertonic sodium chloride on intracranial pressure in patients with acute liver failure. Hepatology 2004; 39:464-470.

10. Davenport A, Will EJ, Davison AM. Early changes in intracranial pressure during haemofiltration treatment in patients with grade 4 hepatic encephalopathy and acute oliguric renal failure. Nephrol Dial Transplant 1990; 5:192-198.

11. Monchi M, Berghmans D, Ledoux D, Canivet JL, Dubois B, Damas P. Citrate vs. heparin for anticoagulation in continuous venovenous hemofiltration: a prospective randomized study. Intensive Care Med 2004;30:260-265. 
12. Morgera S, Scholle C, Voss G, Haase M, Vargas-Hein O, Krausch D, Melzer C, Rosseau S, Zuckermann-Becker H, Neumayer HH. Metabolic complications during regional citrate anticoagulation in continuous venovenous hemodialysis: single-center experience. Nephron Clin Pract 2004;97:c131-c136.

13. Bagshaw SM, Laupland KB, Boiteau PJ, Godinez-Luna T. Is regional citrate superior to systemic heparin anticoagulation for continuous renal replacement therapy? A prospective observational study in an adult regional critical care system. J Crit Care 2005;20:155-161.

14. De Vos J, Hombrouckx R. Citrate anticoagulation and adverse events. Edtna Erca J 2003;29:112-113, 117.

15. Hofmann RM, Maloney C, Ward DM, Becker BN. A novel method for regional citrate anticoagulation in continuous venovenous hemofiltration (CVVHF). Ren Fail 2002; 24:325-335.

\section{Author Affiliations}

Joshua E. Medow, MD*; Shalin R. Sanghvi, $M D^{\prime}$;

R. Michael Hofmann, $M D^{+}$

*Department of Neurosurgery, University of Wisconsin,

Madison, WI USA

Department of Medicine, Section of Nephrology,

University of Wisconsin, Madison, WI USA 